\title{
Chapter 26 \\ The TNO In Vitro Model of the Colon (TIM-2)
}

\author{
Koen Venema
}

\begin{abstract}
This contribution describes the development and use of the TNO in vitro model of the colon (TIM-2). The unique features of this system are briefly discussed, as well as how these contribute to the predictability of this validated in vitro model for clinical trials. Several examples are provided of data where experiments in TIM-2 yielded similar data as clinical trials. Other examples highlight the use of the system to screen dietary components for certain functionality (e.g., increase in butyrate production), or determine likely mechanisms of action of dietary substrates (e.g., the bifidogenic nature of dietary carbohydrates). Moreover, examples are provided of metabolism of dietary compounds such as polyphenols of which the determination of in vivo metabolism is extremely difficult, due to the limited access we have to the colon. The system is inoculated with a microbiota originating from healthy volunteers (single donor or pooled microbiota) of different age, or from people with a disease (e.g., inflammatory bowel disease). Also, differences between the microbiota from lean and obese individuals have been studied. Recent developments in building hypotheses on the role of the microbiota in health and disease have been generated using substrates that were labeled with a stable isotope (i.e., ${ }^{13} \mathrm{C}$ ), that allows tracing the label into microbial biomass and into metabolites produced by the gut microbiota. This has significantly advanced our knowledge on the role of the activity of the gut microbiota in health and disease and the members of the microbiota that are involved in this.
\end{abstract}

Keywords In vitro model $\bullet$ Colon $\bullet$ Microbiota $\bullet$ SCFA $\bullet$ Prebiotics

\subsection{Description of TIM-2}

This contribution focuses on food-application of TIM-2. Although numerous examples are available for pharmaceutical applications as well, these will not be exemplified here.

K. Venema $(\bowtie)$

Beneficial Microbes Consultancy, Wageningen, The Netherlands

e-mail: koen.venema@outlook.com 


\subsubsection{History of the Model}

The TNO computer-controlled, dynamic in vitro gastro-Intestinal Model of the colon (nick-named TIM-2; Fig. 26.1) was developed by TNO some 15 years ago (Minekus et al. 1999). It is based on the TIM-1 system (the TNO in vitro gastrointestinal model of the stomach and small intestine) that is discussed elsewhere in this book.

Briefly, the model consists of four interconnected glass compartments, with a flexible membrane inside (Fig. 26.1a). In between the glass jacket and the membrane there is water of body temperature $\left(37^{\circ} \mathrm{C}\right.$ for humans, $39^{\circ} \mathrm{C}$ for pigs, $41^{\circ} \mathrm{C}$ for birds, etc.). The temperature is controlled by a temperature sensor (Fig. 26.1j). By applying pressure on the water at regular intervals and in a certain sequence, the flexible membrane contracts and causes peristaltic waves which mixes the luminal contents and moves it through the system. This mixing is better than can be accomplished in a fermentor, where phase separation of fluids and solids occur. The system is kept at a $\mathrm{pH}$ of 5.8, the $\mathrm{pH}$ occurring in the proximal colon, or above by continuous measurement of the $\mathrm{pH}$ (Fig. 26.1b) and secretion of $1 \mathrm{M} \mathrm{NaOH}$ (Fig. 26.1c) to neutralize the

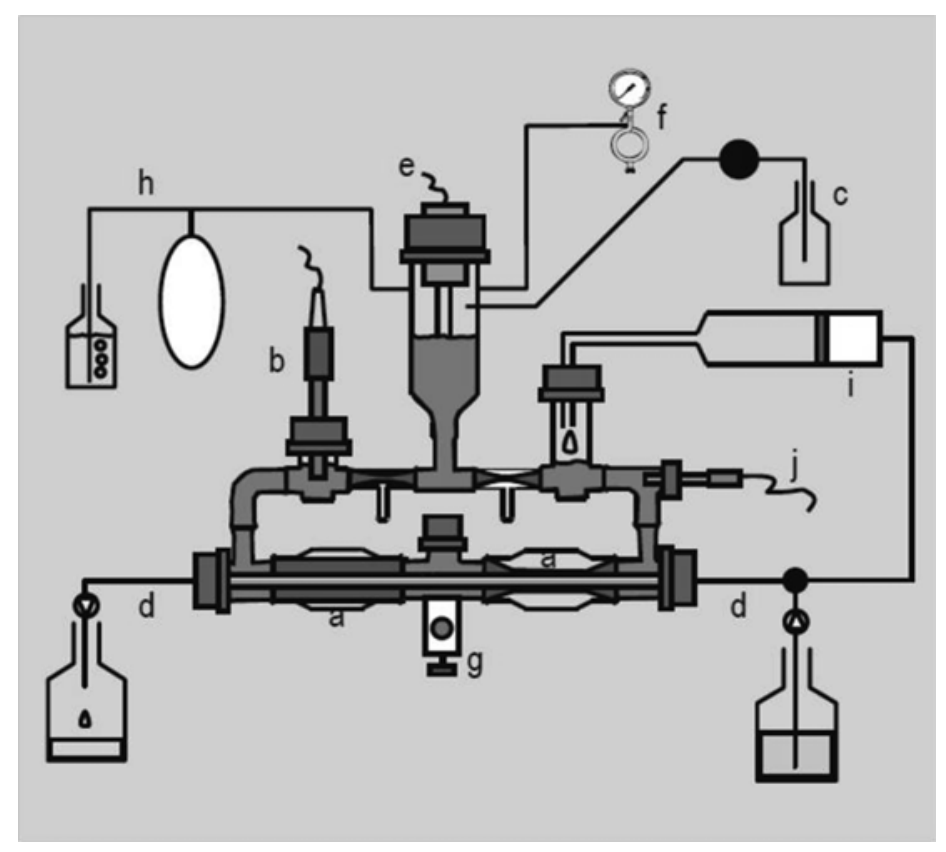

Fig. 26.1 Schematic figure of the TNO in vitro model of the colon (TIM-2). (a) peristaltic compartments containing faecal matter; (b) pH electrode; (c) alkali pump; (d) dialysis liquid circuit with hollow fibre membrane; (e) level sensor; (f) $\mathrm{N}_{2}$ gas inlet; (g) sampling port; (h) gas outlet; (i) 'ileal efflux' container; $(\mathrm{j})$ temperature sensor 
acids that are produced by the microbiota. To prevent accumulation of microbial metabolites, which would lead to the inhibition or death of the microbes in the model when they would accumulate, the system is equipped with a dialysate system, consisting of a bottle with dialysate, a dialysis membrane running through the model and a bottle collecting the spent dialysate (Fig. 26.1d). This dialysis system is unique, and moreover, required to maintain an active microbiota for periods for up to 3 weeks. This was the longest the system has been tested. Normally experiments are performed over a period of 1 week (see Sect. 26.2).

The dialysis system has been tuned to keep physiological concentrations of microbial metabolites in the lumen of the model. For example, short-chain fatty acid (SCFA) concentrations are within the physiological range of $80-120 \mathrm{mM}$ (Venema et al. 2000). The model is equipped with a level sensor to control the volume (Fig. 26.1e). When the volume in the system rises due to addition of the feed or through dialysis, this sensor activates the dial-out pump and maintains the volume at a constant level of $\sim 120 \mathrm{~mL}$. To keep the model anaerobic, it is flushed with gaseous nitrogen (Fig. 26.1f). This, plus the activity of the microbiota in the system, keeps the redox-potential at a level of $\sim-300 \mathrm{mV}$, which is the value that has been reported to occur in the large intestine. Samples can be taken from the lumen through the sampling-port (Fig. 26.1g) or from the spent dialysate (Fig. 26.1d). This allows for a complete mass-balance to be calculated. Gases produced by the microbiota can be sampled too, although normally this is not done and the gas just escapes from the model through a bubbling-vial (Fig. 26.1h). Frequent sampling over time allows the study of the kinetics of production of certain microbial metabolites (e.g., SCFA) on specific substrates. It is obvious that such sampling is not possible in vivo, and hence TIM-2 allows studying the mode of action of certain compounds (foods or drugs) which cannot be studied in detail in man, nor in animals. The microbiota in the system is fed through a food syringe (Fig. 26.1i) which contains a simulated ileal efflux medium (SIEM), which in composition mimics the components that reach the colon from the terminal ileum through the ileal-cecal valve. It consists of some complex carbohydrates, some protein (not all protein is digested in the small intestine [SI]), some residual bile (not all bile is resorbed in the SI), and some minerals and vitamins [for details see Minekus et al. 1999; van Nuenen et al. 2003; Venema et al. 2000].

\subsubsection{Special Features of the Model}

Several features in TIM-2 are unique over other models. First of all, the peristaltic movements of the flexible membrane give a better mixing and movement of components through the entire model than would be accomplished by stirring (in a fermentor) or shaking (on a rocking-platform or otherwise). In TIM-2 there is no phase-separation of solids and liquids, which does occur in other systems. Viscous 'meals' or insoluble components can be used without problems in the system. Secondly, as eluded to above, the dialysis system is not only unique, but also required 
to maintain a highly active microbiota with a similar density as that found in the human large intestine. In batch incubations or less sophisticated models the microbiota is usually inoculated at a much lower density (100-fold or more) and is allowed to slowly grow to physiological densities. However, the metabolites produced by the microbiota start to inhibit further fermentation at these high densities in these systems. Since these metabolites are also taken up by the epithelial cells of the colon (colonocytes) in vivo, TIM-2 mimics much better the physiological situation in the large intestine. In fact, since all metabolites are collected and can be measured, a(n almost) complete mass-balance can be made, which is not possible in vivo, not even in animals, although in scientific studies usually animal are sacrificed to sample as much as possible, including blood samples. However, even in animals a massbalance is not possible, as e.g., butyrate-one of the SCFA—is used by the colonocytes and does not reach the blood circulation. Therefore, TIM-2 allows studying (molecular) mechanisms. This is certainly the case if labeled substrates are used, as will be discussed in other sections of this chapter.

The model is incubated by using a fecal donation from volunteers. This fecal donation can be used in two ways: (a) a fecal donation from individual one can be introduced into one of the TIM-2 systems, the donation from individual 2 in a second unit, and so on. The composition and activity of the microbiota of the individuals can then be compared on say one and the same substrate. This has for instance been done for lactulose, with ten donors (Venema et al. 2003); or (b) the fecal donations of several donors are mixed to create a standardized microbiota that can subsequently be used for $\sim 100$ experiments. This allows comparison of multiple substrates or conditions starting with the same microbiota composition. This pool of fecal donations to a certain extent mimics a (small) population. It has been argued by many that mixing different fecal samples may disturb the microbial balance within a single fecal sample, but as far as we know, no direct comparison has been done to show this. Recently, we have set out to show that by mixing a number of different fecal donations, the functionality of the microbiota is not influenced (Aguirre et al. 2014). That is, the individual microbiotas showed the same functionality as the pool and produced very similar amounts and ratios of microbial metabolites (amongst others SCFA), despite being different in microbial composition. This underscores our hypothesis that there is an enormous functional overlap between microbes in the large intestine. This is not entirely surprising, as there are only a limited number of biochemical routes (let's say 5) from e.g., glucose to acetate. Since the microbiota is composed of approximately 200 species or more, there has to be enormous functional overlap between these microorganisms.

Upon introduction of the microbiota in the system, an adaptation period of $\sim 16 \mathrm{~h}$ is applied, to allow the microbes to adapt to their new environment and the feed components. After that the experimental period starts, which normally takes $72 \mathrm{~h}$ (see Sect. 26.2). Fecal donations can be obtained from healthy volunteers of different age-classes (baby, adults, elderly), people with a disease or disorder [e.g., inflammatory bowel disease; (van Nuenen et al. 2004)], or from lean vs. obese individuals. 


\subsubsection{Stability and Reproducibility of the System}

Since the model is computer-controlled it is highly reproducible. This has been shown e.g., by the clustering of the microbiota after the adaptation period referred to in the previous section (Fig. 26.2). In this example, the microbiotas originating from lean and obese individuals were compared. The figure shows that both microbiotas clustered separately, but that each $\mathrm{t}=0$ sample of the lean microbiota clustered very closely together with the other lean samples, and similarly for the obese samples. It should be said that the microbiota in this adaptation period undergoes a change in composition, as it adapted to the model and feed (Rajilic-Stojanovic et al. 2010). This is not entirely surprising, as the conditions in the model are different to the colon, although we try to simulate them as good as possible. But, the system does not contain epithelial cells or immune cells that may influence the microbiota composition. This will be discussed in more detail later (see Sect. 26.5).

No matter whether the system is inoculated by a standardized microbiota or a microbiota from a single donor, it is highly reproducible. This has been shown in numerous studies (Kovatcheva-Datchary et al. 2009; Martinez et al. 2012; Rose et al. 2010). This is why experiments in TIM-2 are performed in duplicate.

In every project a control with the standard growth medium (SIEM) is performed. The other conditions applied in such projects are then compared to the effects obtained with the standard medium. This allows correcting for effects that occur simply through use of the in vitro model.

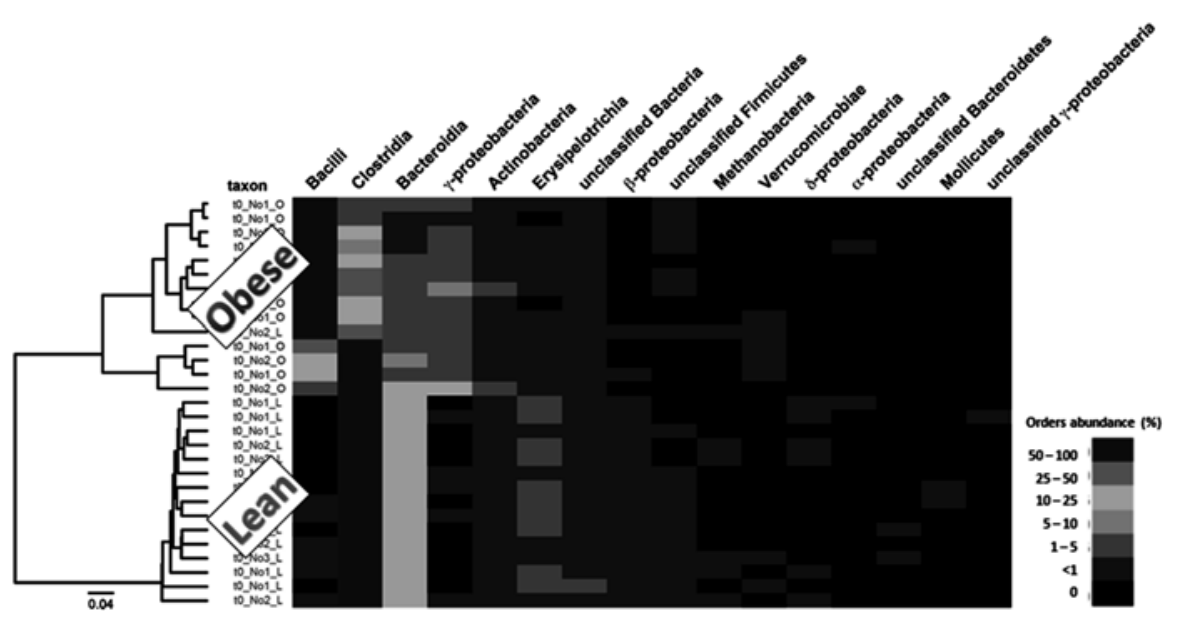

Fig. 26.2 Phylogentic tree (left) and heatmap (right) of samples from TIM-2 taken at $\mathrm{t}=0$ in an experiment where a lean and obese microbiota was compared. The lean and obese microbiotas cluster separately, although all $t=0$ samples of each microbiota (lean or obese) cluster closely together 


\subsubsection{Relevance to Human In Vivo Situation}

The model has been developed and optimized with the use of data from suddendeath individuals (Macfarlane et al. 1992). The data from these samples with respect to microbial metabolites and microbiota composition were used to develop TIM-2.

The effect of several food components that have been well-established in vivo have been confirmed in TIM-2. These include the bifidogenic nature of inulin (van Nuenen et al. 2003) and galacto-oligosaccharides (GOS) (Maathuis et al. 2012), or the high production of butyrate out of resistant starches (Rose et al. 2010). Due to this, the validated system is frequently used to test experimental substrates and is thought to be predictive for the in vivo situation.

Yet, in some situations the model is even capable of predicting results in the proximal colon that cannot be measured in a clinical trial. This has for instance been done in experiments using lactulose (Venema et al. 2003). Here, fecal donations were obtained from volunteers before they ingested lactulose for 10 days, and after. Both fecal donations were inoculated independently in TIM-2 and were fed lactulose in the system. The production of the major microbial metabolites, the SCFA, was analyzed both in fecal samples as well as in TIM-2 samples. At first surprisingly, the in vivo fecal samples before and after lactulose did not differ in SCFA content and ratio, while the samples in TIM- 2 showed a marked reduction in butyrate production. However, since lactulose is a disaccharide, it is fermented very quickly by the gut microbiota (as also indicated by the accumulation of lactate), and this occurs in the proximal colon. We now hypothesize that the SCFA that were produced by the microbiota in vivo in the proximal colon were all taken up during transit to the distal colon, and that the SCFA present in the excreted fecal samples reflect those produced locally in the distal colon, rather than reflect the SCFA that were produced in the proximal colon. This is highly likely as it has been estimated that $95 \%$ of the SCFA produced by the microbiota are absorbed. Therefore, TIM-2 gave insight in the processes at the site where the fermentation of lactulose happened-the proximal colon. This is data that could not be inferred from fecal samples taken from these volunteers.

\subsubsection{Quality in Relation to Other Models with the Same Applicability}

Compared to other in vitro models mimicking the colon, TIM-2 has a number of features that are unique (see Sect. 26.1.2) and that allow to predict what would happen in a clinical trial. In the previous section the example of lactulose was given. To validate these results one would need to sample the proximal colon, which is only possible when using a long catheter that is inserted through the nose or throat and reaches the proximal colon (Venema 2011), or a tube that is stuck up the rectum to reach the proximal part of the large intestine. Although this has been done, it is 
very invasive and it is difficult to get ethical approval for testing functionality of numerous food components. Nevertheless, it is our belief that the results that were found in TIM-2 predict what happens in vivo, even though this could not be confirmed because the analytes in fecal matter in this case were not representative for those produced in the proximal colon, as they had all been absorbed by the colonic tissue during transit from proximal to distal colon, which may take anything from 24 to $72 \mathrm{~h}$.

One of the reasons the system can predict what happens in real life so well is the presence of the dialysis system. As discussed above, this system prevents the accumulation of microbial metabolites, which normally are also taken up by the gut epithelium. In other systems metabolites tend to accumulate. Apart from slowing down or stopping further microbial breakdown of the substrates under study (Ramasamy et al. 2014), both the kinetics and true production of e.g., SCFA are inaccurate in these incubations. Since the metabolites are not removed, this allows for them to be converted into one another. For example, two molecules of acetate can be coupled to form butyrate, while lactate can be converted to propionate or butyrate. While this normally occurs to a certain extent in the gut microbiota, and is called cross-feeding (Kovatcheva-Datchary et al. 2009), in batch incubations this happens to a degree that is no longer physiological.

Also, the accumulation of the SCFA (in particular) leads to the inhibition of certain members of the microbiota, certainly if this is accompanied by a drop in $\mathrm{pH}$. Because of this, the composition of the microbiota may change and no longer reflect that of the normal gut. It should be said that even in TIM-2 the microbiota will change after introduction of the inocula (Rajilic-Stojanovic et al. 2010).

Due to the fact that the system closely mimics physiological parameters, the experiments in TIM-2 usually take 1 week (see Sect. 26.2). In contrast, other models that mimic the large intestine usually take several weeks due to the need to reach steady state. Since multiple units of TIM-2 can be run at the same time (maximally 10), comparisons with a control can be made and steady state in TIM-2 is not strictly required. For a more extensive review of TIM-2 in comparison to other in vitro models mimicking the colon, please refer to Venema and van den Abbeele (2013).

\subsection{General Protocol}

After introduction of the microbiota into TIM-2 (at $\sim 11 \% \mathrm{w} / \mathrm{w} ; 120 \mathrm{~mL}$ ), the microbiota is allowed to adapt to the new situation over a period of $16 \mathrm{~h}$ and is fed with the standard SIEM medium, composed of complex carbohydrates, protein, some ox-bile, Tween 80 and vitamins and minerals (Maathuis et al. 2012). A total of $60 \mathrm{~mL} /$ day of this medium is fed to the microbiota. The medium is provided at a speed of $2.5 \mathrm{~mL} / \mathrm{h}$, reflecting the occasional opening and closing of the ileal-cecal valve in vivo.

After the $16 \mathrm{~h}$ adaptation period, there usually is a $2-4 \mathrm{~h}$ starvation period to allow the microbiota to ferment all the available carbohydrates in the system. 
After this, the standard medium is replaced by the test carbohydrate. This test medium is fed for another $72 \mathrm{~h}$. Just prior to the start of feeding the test medium, samples are taken from the lumen and dialysate at $\mathrm{t}=0$. Subsequently, every $24 \mathrm{~h}$ samples are taken from both the lumen and dialysate, and after analysis the cumulative production of microbial metabolites is calculated. Similarly, samples are taken every $24 \mathrm{~h}$ from the lumen to study changes in microbiota composition using microarray or, nowadays, next generation sequencing technology.

Although the above is considered to be a general protocol, every project may require the tailor-made adjustment of this protocol. Some experiments may not use a carbohydrate test substrate, but e.g. polyphenols (Gao et al. 2006; Bordonaro et al. 2014) or saponins (Kong et al. 2009), which requires addition of these compounds to the standard medium.

Over the past 5 years or so, we have also ran experiments in which we gave a single shot of $1 \mathrm{~g}$ of uniformly stable-isotope $\left({ }^{13} \mathrm{C}\right)$ labeled carbohydrates at $\mathrm{t}=0$ and then followed degradation of these substrates over time. Incorporation of label into microbial biomass using Stable Isotope Probing (SIP) (Maathuis et al. 2012) and into microbial metabolites using NMR and LC-MS (Binsl et al. 2010) were used to trace the fate of these labeled carbohydrates.

By choice no mucin is added to the system. The only commercial source of mucin is pig gastric mucin, which has a completely different composition than human colonic mucin, and therefore is not a good mimic for human colonic mucin. Therefore, the choice was made not to standardly add mucin to TIM-2 fermentations. In cases where addition of mucin would be essential, it could be added, although the above should be taken into consideration. Similarly, we do not add the insoluble and unfermentable cellulose. It is hardly fermentable to the human gut microbiota (Slavin et al. 1981), and microorganisms capable of using this substrate appear to only be present in fecal samples from methane-excretors while this community remained undetectable in non-methane-excretors (Robert and Bernalier-Donadille 2003). Sometimes however, it is used as a negative (non-fermentable) control.

Most of the times, the model simulates the proximal colon with a $\mathrm{pH}$ of 5.8, although sometimes the systems mimics the entire colon and the $\mathrm{pH}$ is programmed as a gradient over time from 5.8 in the proximal colon, to 6.4 in the transverse colon, to 7.0 in the distal colon. The lumen is then considered a plug which transits through the different parts of the large intestine.

\subsection{Controls to Test Stability and Performance of the Model}

TIM-2 is completely computer-controlled. All data with respect to the secretion of fluids (feed, $\mathrm{NaOH}$, dialysate) as well as temperature and $\mathrm{pH}$ are monitored and stored. Moreover, these values are plotted on a computer-screen, such that the person operating the model is immediately aware of how the system is performing. Furthermore, the stability and performance of the system is monitored by studying these values after the experiment, in addition to monitoring the production of SCFA 
under standardized conditions. The production of SCFA should essentially be equal under identical conditions even if the experiments are performed over a stretch of several months. Unfortunately, SCFA cannot be measured online (yet), but the secretion of $\mathrm{NaOH}$ (to neutralize the drop in $\mathrm{pH}$ caused by the acids) is used as a proxy for SCFA production and this has worked very well to monitor the performance of the model. When a parameter is out of range, the run is terminated. Usually this happens because of a mechanical error in a pump or due to a leak in the system allowing oxygen to diffuse in, which would preclude a complete anaerobic environment.

The $\mathrm{pH}$ may not drop below 5.8 (the set-point value), but is allowed to rise above this value if for instance protein fermentation is expected or occurs. Protein fermentation leads to the production of putrefactive metabolites, amongst others ammonia, which raises the $\mathrm{pH}$ in the system. Under circumstances of excess protein it may in fact raise to a $\mathrm{pH}$ near neutrality.

The speed of dialysis is important to maintain physiological levels of microbial metabolites in the system. If dialysis does not occur (e.g., a pump broke down, or a tube got disconnected) this is immediately reflected in the production of acids (with $\mathrm{NaOH}$ as the proxy for that) and eventually in the microbiota composition, although the latter is only established days to weeks after the experiments, when the molecular analyses have been done.

Usually the experiments are very reproducible. The standard deviation (or range) normally falls within $10 \%$. This is why it was decided in the early 2000 s to only perform duplicate experiments, and only repeat an experiment if the duplicates were way off.

\subsection{Read-Out of the System and How This Information Can Be Used}

Because of the health benefit of SCFA (den Besten et al. 2013), in particular butyrate (Havenaar 2011), most of the experiments carried out in the past 15 years focused on carbohydrate fermentation and the consequent production of the individual SCFA and their ratio. Experiments could screen numerous substrates for optimal SCFA production or would advance pre-established hypotheses that certain compounds would lead to the production of increased levels of certain SCFA, such as the case for butyrate when starch is fermented.

This requires the analysis and calculation for the cumulative production of these SCFA over time. Since all metabolites that are produced by the microbiota are collected in the system (from dialysate and lumen) this assessment of total SCFA production can be made.

Coupled to analyses for SCFA the (change in) composition of the microbiota is usually measured, to establish the link between SCFA produced and the microorganisms responsible for this. With the advent of the stable-isotope technology in gut microbiology this has become even easier, as there is a direct link between label incorporation into metabolites and into microbial biomass (Venema 2011). 
However, the system has been used for numerous other applications as well. For instance, the effect of probiotics upon antibiotic treatment to quickly re-establish the microbial balance (Rehman et al. 2012), fermentation of polyphenols from tea, citrus or chocolate into more simple phenolic compounds, each with their own functionality (Bordonaro et al. 2014; Gao et al. 2006), or the metabolism of saponins from traditional Chinese medicines (Kong et al. 2009). No matter what the example, the aim has always been to increase the health of the host through the microbiota. Recently, the microbiota has also been shown to be involved in obesity (Venema 2010). This has led to the investigation in TIM-2 of the metabolism of microbiotas originating from lean and obese individuals (as yet unpublished). Numerous attempts are made to mine the microbiota for functional (healthy) activities (Roeselers et al. 2012). It is expected that TIM-2 may play a role in deciphering the beneficial microbes and metabolites that play a role in health and disease.

Sometimes samples from TIM- 2 are incubated with other in vitro models described in this book, such as Caco-2 cell cultures (Lamers et al. 2003), immune cells (van Nuenen et al. 2005), or ex vivo pig intestinal tissue. In these cases, the effects of the microbial metabolites on parameters studied in these cells are of importance, such as barrier function, DNA damage, or induction of satiety hormones (such as GLP-1 or PYY). We have recently shown that samples from TIM-2 induce PYY, which is involved in appetite regulation, in pig intestinal tissue (Bussolo et al. 2014).

\subsection{Advantages, Disadvantages and Limitations of the System}

TNO has 10 units of TIM-2 available, allowing multiple parameters to be tested in parallel. In contrast to other multi-compartmental in vitro models, experiments are quick (usually three test days or less), yet still physiological and predictive. The advantages over other models are the presence of peristaltics and a dialysis system, the latter of which allows production of physiological concentrations of metabolites, and a highly active microbiota of normal density to be used. Another advantage is that a single parameter in the system can be changed, and the effect of that single parameter on microbiota activity can be studied. This has been done e.g., when studying the effect of different $\mathrm{pH}$ 's on fermentation of carbohydrates (unpublished). Naturally, in vitro models have their limitations. As with every other in vitro model that mimics the colon, TIM-2 does not have epithelial or immune cells. However, as discussed in Sect. 26.4, samples can be incubated with these cells for even better predictability. Another limitation is that the model has been developed on the basis of literature data of mostly health individuals. Due to this, it is unclear exactly which parameters to simulate when simulating patient populations as discussed in van Nuenen et al. (2004). Another limitation is that (apart from volume and $\mathrm{pH}$ ) there are no feed-back mechanisms in the system. Therefore, the experiments in such in vitro models will always be at most an indication of what may occur in real life, and the results need to be interpreted with care. 
Open Access This chapter is distributed under the terms of the Creative Commons Attribution Noncommercial License, which permits any noncommercial use, distribution, and reproduction in any medium, provided the original author(s) and source are credited.

\section{References}

Aguirre A, Ramiro-Garcia J, Koenen ME, Venema K (2014) To pool or not to pool? Impact of the use of individual and pooled faecal samples for in vitro fermentation studies. J Microbiol Methods 107:1-7

Binsl TW, De Graaf AA, Venema K, Heringa J, Maathuis A, De Waard P, Van Beek JH (2010) Measuring non-steady-state metabolic fluxes in starch-converting faecal microbiota in vitro. Benefic Microbes 1:391-405

Bordonaro M, Venema K, Putri AK, Lazarova D (2014) Approaches that ascertain the role of dietary compounds in colonic cancer cells. World J Gastrointest Oncol 6:1-10

Bussolo CS, Roeselers G, Troost F, Jonkers D, Koenen ME, Venema K (2014) Prebiotic effects of cassava bagasse in TNO's in vitro model of the colon (TIM-2) in lean versus obese microbiota. J Funct Foods 11:210-220

den Besten G, van Eunen K, Groen AK, Venema K, Reijngoud DJ, Bakker BM (2013) The role of short-chain fatty acids in the interplay between diet, gut microbiota, and host energy metabolism. J Lipid Res 54:2325-2340

Gao K, Xu A, Krul C, Venema K, Liu Y, Niu Y, Lu J, Bensoussan L, Seeram NP, Heber D, Henning SM (2006) Of the major phenolic acids formed during human microbial fermentation of tea, citrus, and soy flavonoid supplements, only 3,4-dihydroxyphenylacetic acid has antiproliferative activity. J Nutr 136:52-57

Havenaar R (2011) Intestinal health functions of colonic microbial metabolites: a review. Benefic Microbes 2:103-114

Kong H, Wang M, Venema K, Maathuis A, van der Heijden R, van der Greef J, Xu G, Hankemeier $\mathrm{T}$ (2009) Bioconversion of red ginseng saponins in the gastro-intestinal tract in vitro model studied by high-performance liquid chromatography-high resolution Fourier transform ion cyclotron resonance mass spectrometry. J Chromatogr A 1216:2195-2203

Kovatcheva-Datchary P, Egert M, Maathuis A, Rajilic-Stojanovic M, de Graaf AA, Smidt H, de Vos WM, Venema K (2009) Linking phylogenetic identities of bacteria to starch fermentation in an in vitro model of the large intestine by RNA-based stable isotope probing. Environ Microbiol 11:914-926

Lamers RJ, Wessels EC, van de Sandt JJ, Venema K, Schaafsma G, van der Greef J, van Nesselrooij $\mathrm{JH}$ (2003) A pilot study to investigate effects of inulin on Caco-2 cells through in vitro metabolic fingerprinting. J Nutr 133:3080-3084

Maathuis AJ, van den Heuvel EG, Schoterman MH, Venema K (2012) Galacto-oligosaccharides have prebiotic activity in a dynamic in vitro colon model using a (13)C-labeling technique. J Nutr 142:1205-1212

Macfarlane GT, Gibson GR, Cummings JH (1992) Comparison of fermentation reactions in different regions of the human colon. J Appl Bacteriol 72:57-64

Martinez RC, Cardarelli HR, Borst W, Albrecht S, Schols H, Gutierrez OP, Maathuis AJ, de Melo Franco BD, De Martinis EC, Zoetendal EG, Venema K, Saad SM, Smidt H (2012) Effect of galactooligosaccharides and Bifidobacterium animalis $\mathrm{Bb}-12$ on growth of Lactobacillus amylovorus DSM 16698, microbial community structure, and metabolite production in an in vitro colonic model set up with human or pig microbiota. FEMS Microbiol Ecol 84:110-123

Minekus M, Smeets-Peeters M, Bernalier A, Marol-Bonnin S, Havenaar R, Marteau P, Alric M, Fonty G, Huis in't Veld JH (1999) A computer-controlled system to simulate conditions of the large intestine with peristaltic mixing, water absorption and absorption of fermentation products. Appl Microbiol Biotechnol 53:108-114 
Rajilic-Stojanovic M, Maathuis A, Heilig HG, Venema K, de Vos WM, Smidt H (2010) Evaluating the microbial diversity of an in vitro model of the human large intestine by phylogenetic microarray analysis. Microbiology 156:3270-3281

Ramasamy US, Venema K, Schols HA, Gruppen H (2014) The effect of soluble and insoluble fibers within the in vitro fermentation of chicory root pulp by human gut bacteria. J Agric Food Chem 62(28):6794-6802

Rehman A, Heinsen FA, Koenen ME, Venema K, Knecht H, Hellmig S, Schreiber S, Ott SJ (2012) Effects of probiotics and antibiotics on the intestinal homeostasis in a computer controlled model of the large intestine. BMC Microbiol 12:47

Robert C, Bernalier-Donadille A (2003) The cellulolytic microflora of the human colon: evidence of microcrystalline cellulose-degrading bacteria in methane-excreting subjects. FEMS Microbiol Ecol 46:81-89

Roeselers G, Bouwman J, Venema K, Montijn R (2012) The human gastrointestinal microbiota-an unexplored frontier for pharmaceutical discovery. Pharmacol Res 66:443-447

Rose DJ, Venema K, Keshavarzian A, Hamaker BR (2010) Starch-entrapped microspheres show a beneficial fermentation profile and decrease in potentially harmful bacteria during in vitro fermentation in faecal microbiota obtained from patients with inflammatory bowel disease. $\mathrm{Br} \mathrm{J}$ Nutr 103:1514-1524

Slavin JL, Brauer PM, Marlett JA (1981) Neutral detergent fiber, hemicellulose and cellulose digestibility in human subjects. J Nutr 111:287-297

van Nuenen MHMC, Meyer PD, Venema K (2003) The effect of various inulins and Clostridium difficile on the metabolic activity of the human colonic microbiota in vitro. Microb Ecol Health Dis 15:137-144

van Nuenen MH, Venema K, van der Woude JC, Kuipers EJ (2004) The metabolic activity of fecal microbiota from healthy individuals and patients with inflammatory bowel disease. Dig Dis Sci 49:485-491

van Nuenen MH, de Ligt RA, Doornbos RP, van der Woude JC, Kuipers EJ, Venema K (2005) The influence of microbial metabolites on human intestinal epithelial cells and macrophages in vitro. FEMS Immunol Med Microbiol 45:183-189

Venema K (2010) Role of gut microbiota in the control of energy and carbohydrate metabolism. Curr Opin Clin Nutr Metab Care 13:432-438

Venema K (2011) Stable isotope probing and the human gut. In: Murrell JC, Whiteley AS (eds) Stable isotope probing and related technologies. ASM Press, Washington, DC, pp 233-257

Venema K, van den Abbeele P (2013) Experimental models of the gut microbiome. Best Pract Res Clin Gastroenterol 27:115-126

Venema K, van Nuenen HMC, Smeets-Peeters M, Minekus M, Havenaar R (2000) TNO's in vitro large intestinal model: an excellent screening tool for functional food and pharmaceutical research. Ernährung/Nutrition 24:558-564

Venema K, van Nuenen MHMC, van den Heuvel EG, Pool W, van der Vossen JMBM (2003) The effect of lactulose on the composition of the intestinal microbiota and short-chain fatty acid production in human volunteers and a computer-controlled model of the proximal large intestine. Microb Ecol Health Dis 15:94-105 